\title{
Consequência do desmame precoce para a criança
}

\author{
Consequence of early weaning for the child \\ Consecuencia del destete temprano para el niño
}

Vitoria Vilas Boas da Silva Bomfim

ORCID: https://orcid.org/0000-0003-4897-0279

Centro Universitário Jorge Amado, Brasil E-mail: pesquisaclinica9@gmail.com

Maria Clara Teles Cabanelas Macedo

ORCID: https://orcid.org/0000-0003-2025-7789

Universidade Salvador, Brasil

E-mail: claracabanelas@gmail.com

Iara Neves Vieira Cavalcante

ORCID: https://orcid.org/0000-0001-8645-6487 Universidade Federal da Bahia, Brasil

E-mail: iara.cavalcante@ufba.br

André Kelves da Silva Araujo

ORCID: https://orcid.org/0000-0003-3192-2166

Christus Faculdade do Piauí, Brasil

E-mail: andrekelves008@gmail.com

Ianka Catarino Mourao de Sousa

ORCID: https://orcid.org/0000-0002-4924-4319

Universidade Federal do Maranhão, Brasil

E-mail: iankamourao12@gmail.com

Maria Eduarda Wanderley de Barros Silva

ORCID: https://orcid.org/0000-0002-4642-3282

Universidade Federal de Campina Grande, Brasil

E-mail: eduarda.wanderley@ outlook.com

Joanna Luviter Santos Pedreira

ORCID: https://orcid.org/0000-0002-4896-6985

Universidade Salvador, Brasil

E-mail: jluviter22@gmail.com

Jéssica de Freitas Soares

ORCID: https://orcid.org/0000-0002-4252-3464

Universidade Federal de Campina Grande, Brasil

E-mail: jessicafse@ @otmail.com

Andressa Fernandes da Silva

ORCID: https://orcid.org/0000-0002-1942-0860

Instituto Nacional de Cardiologia, Brasil

E-mail: andressafdurso@gmail.com

Vitória Cristine Pereira da Silva

ORCID: https://orcid.org/0000-0003-4256-4253

Universidade Salvador, Brasil

E-mail: vitoriacps1@outlook.com

Mirella Rodrigues Evaristo

ORCID: https://orcid.org/0000-0001-8086-8376 Centro Universitário da Vitória de Santo Antão, Brasil

E-mail: mirellapsicologa2018.2@gmail.com

Janaina Oliveira Silva

ORCID: https://orcid.org/0000-0002-0620-865X

Faculdade Venda Nova do Imigrante, Brasil

E-mail: enfermeira.janainaoliveira@gmail.com

Caroline Cristina de Oliveira Cunha

ORCID: https://orcid.org/0000-0003-3985-5746

Escola Superior Madre Celeste, Brasil

Resumo

E-mail: caroline.ccunha@gmail.com

Objetivo: E identificar conforme a literatura científica quais as repercussões do desmame precoce para a criança. Metodologia: Trata-se de uma revisão integrativa, os dados foram extraídos da Biblioteca Virtual de Saúde e no Google Acadêmico. Foram incluídos os artigos disponíveis na integra, no idioma português, inglês e espanhol, no período de 2015-2020, e excluídos os artigos duplicados e que não atendem a questão de pergunta. A pesquisa foi constituída por 4 artigos. Resultados: Algumas consequências foram identificadas: diarreia, mortalidade e morbidade infantil, alergias, obesidade, desnutrição, infecções respiratórias graves e desenvolvimento motor-oral incompleto. 
Considerações Finais: O aleitamento materno completo é importante para a prevenção de doenças infecciosas quanto para o crescimento e desenvolvimento das crianças.

Palavras-chave: Aleitamento materno; Desmame precoce; Consequências.

\begin{abstract}
Objective: And to identify, according to the scientific literature, the repercussions of early weaning for the child. Methodology: This is an integrative review, data were extracted from the Virtual Health Library and Google Scholar. Articles available in full, in Portuguese, English and Spanish, in the period 2015-2020, were included, and duplicated articles that do not meet the question were excluded. The research consisted of 4 articles. Results: Some consequences were identified: diarrhea, infant mortality and morbidity, allergies, obesity, malnutrition, severe respiratory infections and incomplete oral motor development. Final Considerations: Complete breastfeeding is important for the prevention of infectious diseases and for the growth and development of children.
\end{abstract}

Keywords: Breastfeeding; Early weaning; Consequences.

\title{
Resumen
}

Objetivo: E identificar, según la literatura científica, las repercusiones del destete precoz para el niño. Metodología: Se trata de una revisión integradora, los datos fueron extraídos de la Biblioteca Virtual en Salud y Google Scholar. Se incluyeron artículos disponibles en su totalidad, en portugués, inglés y español, en el período 2015-2020, y se excluyeron los artículos duplicados que no cumplieran con la pregunta. La investigación consta de 4 artículos. Resultados: Se identificaron algunas consecuencias: diarrea, mortalidad y morbilidad infantil, alergias, obesidad, desnutrición, infecciones respiratorias severas y desarrollo motor oral incompleto. Consideraciones finales: La lactancia materna completa es importante para la prevención de enfermedades infecciosas y para el crecimiento y desarrollo de los niños.

Palabras clave: Lactancia materna; Destete temprano; Consecuencias.

\section{Introdução}

Considera-se desmame precoce a interrupção do aleitamento materno exclusivo (AME) antes do lactante ter completado seis meses de vida. A introdução de outros tipos de alimentos na dieta da criança tem sido frequente, com consequências potencialmente danosas à saúde do bebê. A Organização Mundial de Saúde (OMS) considera o leite materno um fator determinante no crescimento e desenvolvimento adequado da criança. O leite materno tem na sua composição carboidratos, lipídios, proteínas, minerais, vitaminas e água (Brasil, 2002).

Somente $38,6 \%$ dos bebês brasileiros se alimentam exclusivamente com leite materno nos primeiros cinco meses de vida, segundo a OMS. A taxa é considerada abaixo do ideal, mas regular em relação a outros países. A média mundial de amamentação nos primeiros seis meses de vida fica em torno de $20 \%$ a $40 \%$. No quesito alimentação exclusiva de leite nos primeiros meses, o Brasil está na frente da Argentina (33\%) e atrás da Bolívia (64,3\%). Quando considerada a amamentação até um ano, o índice brasileiro melhora (47\%); até os dois anos, contudo, esse número cai pela metade (26\%) (Brasil, 2017).

Vários fatores podem afetar o início e a manutenção do aleitamento materno exclusivo, a exemplo do grau de escolaridade materna, o retorno da mulher ao mercado de trabalho, o baixo peso da criança ao nascer, além da intenção e a auto eficiência em amamentar. A prematuridade por sua vez, também é um fator condicionante, uma vez que implica na imaturidade fisiológica do recém-nascido quanto ao reflexo de pega e sucção do leite materno, podendo, inclusive, comprometer a produção láctea pela mãe devido à ausência de estímulo. Logo, o nascimento pré-termo implica, ocasionalmente, em prejuízos para a implementação e manutenção do AME (ARQ. Catarinense de MED, 2020).

Diante demostrado sobre o estudo, quais as repercussões do desmame precoce para a criança? E identificar conforme a literatura científica quais as repercussões do desmame precoce para a criança.

\section{Metodologia}

O estudo consiste em uma revisão integrativa, que tem como finalidade sintetizar resultados obtidos em pesquisas sobre um tema ou questão, de maneira sistemática, ordenada e abrangente. É denominada integrativa, pois fornece informações 
mais amplas sobre um assunto/problema, constituindo assim, um corpo de conhecimento (Cordeiro et al. 2007; Prisma Group, 2015).

Após a revisão integrativa dos materiais, que tem como questão de pesquisa e se constitui em "Quais as consequências do desmame precoce para a criança?", buscamos elaborar um texto dissertativo divido em quatro etapas que darão norte a revisão, destacando a importância do aleitamento materno, formas de tratamento para o desmame precoce e suas consequências, a importância da promoção ao aleitamento materno e papel do enfermeiro frente à problemática.

O estudo foi desenvolvido na Biblioteca Virtual em Saúde (BVS), e no Google Acadêmico a partir das bases de dados Medical Literature Analysis and Retrieval System Online (MEDLINE), Literatura Latino-americana e do Caribe em Ciências da Saúde (LILACS) e Base de Dados em Enfermagem (BDENF). Para tanto, os descritores utilizados, "aleitamento materno", "desmame precoce" e "consequências".

Associando a primeira etapa e a segunda, estabelecemos critérios de inclusão e exclusão de estudos. No critério de inclusão: artigos disponíveis na integra nos últimos cinco anos (2015 a 2020) que atendam a questão de pesquisa, nos idiomas português, inglês e espanhol. Serão excluídos: artigos duplicados, trabalhos de conclusão de curso (TCC), dissertações e estudos que não tem relação com o estudo.

Os artigos serão organizados de acordo com os autores, anos de publicações, principais e objetivos de pesquisa e a síntese da pesquisa que dará origem posteriormente a um quadro síntese. Será apresentada a síntese da revisão integrativa, respeitando todas as questões éticas, relacionado à citação dos autores. Os pesquisadores respeitam a autoria das fontes pesquisadas, mencionando as referências bibliográficas e autores, conforme lei que rege os direitos autorais no Brasil (Brasil, 1998).

\section{Resultados e Discussão}

Após a leitura dos artigos apenas quatro se associaram com o tema em estudo. Em relação ao idioma dos artigos selecionados, quatro estavam na língua portuguesa, conforme mostra o Quadro 1.

Com relação ao país de origem dos artigos, todos foram produzidos no Brasil. Os artigos tiveram uma variação entre o período de 2017 a 2020, sendo o ano de 2020 o mais predominante com dois artigos publicados.

Em relação ao periódico de publicação, os artigos foram publicados em uma revista diferentes, sendo em revistas nacionais como a Revista Unimontes Científica, com maior dominância os periódicos vinculados a revistas online e universidades públicas.

Ao analisar os delineamentos de pesquisa mais frequentes nos artigos estudo, observou-se que, três artigos utilizaram a abordagem metodológica qualitativa e um artigo a quantitativa. No que diz ao respeito aos estudos que utilizaram a abordagem metodológica qualitativa, os métodos mais usados foram: descritivo (três artigos) e avaliativo (um artigo).

A partir da exploração dos estudos selecionados, obtiveram comprovação de que as mães não tinham conhecimento sobre as consequências do desmame precoce para a criança. No entanto, algumas consequências ficaram mais evidentes entre os estudos, sendo elas: diarreia (4 artigos) mortalidade infantil (3 artigos), desnutrição (3 artigos), morbidade (3 artigos), problemas respiratórios agudos (1 artigo). Vide Quadro 1. 
Quadro 1 - Resumo dos artigos selecionados para compor a amostra final do estudo.

\begin{tabular}{|c|c|c|c|c|}
\hline ID & Autor & Periódico & Tipo de Estudo & Resultado do Estudo \\
\hline 01 & $\begin{array}{l}\text { Accioly, E. et } \\
a l ., 2017\end{array}$ & Unimontes & $\begin{array}{l}\text { Pesquisa } \\
\text { Explorativa }\end{array}$ & $\begin{array}{l}\text { O desmame precoce pode ser considerado } \\
\text { violência contra a criança, uma vez que a deixa } \\
\text { exposta ao risco de doenças infecciosas, } \\
\text { incidência de alergias, morbidade e mortalidade, } \\
\text { diarreia, infecções respiratórias agudas e } \\
\text { desnutrição. }\end{array}$ \\
\hline 02 & $\begin{array}{l}\text { França, Costa, et } \\
\text { al., } 2020\end{array}$ & Revista Artigo.Com & $\begin{array}{l}\text { Pesquisa } \\
\text { Descritiva }\end{array}$ & $\begin{array}{l}\text { Como maiores propensões a diarreias, alergias } \\
\text { alimentares e desenvolvimento motor-oral } \\
\text { incompleto da criança. }\end{array}$ \\
\hline 03 & Silva et al., 2020 & $\begin{array}{lr}\text { Revista } \quad \text { Pesquisa, } \\
\text { Sociedade } \\
\text { Desenvolvimento }\end{array}$ & $\begin{array}{l}\text { Pesquisa } \\
\text { Descritiva }\end{array}$ & $\begin{array}{l}\text { O desmame precoce é um fator predisponente para } \\
\text { doenças como: desnutrição, diarreia, obesidade } \\
\text { Infantil, entre outros problemas de saúde pública, } \\
\text { além de contribuir para o aumento da mortalidade } \\
\text { infantil. }\end{array}$ \\
\hline 04 & $\begin{array}{l}\text { Figueiredo, JTC } \\
\text { et al., } 2018\end{array}$ & Revista Ciência \& Saberes & $\begin{array}{l}\text { Pesquisa } \\
\text { Descritiva }\end{array}$ & $\begin{array}{l}\text { O desmame precoce é uma das causas de } \\
\text { mortalidade infantil, no primeiro ano de idade, } \\
\text { também considerado como um dos fatores de } \\
\text { doenças em crianças como a desnutrição e diarreia } \\
\text { quando a introdução precoce de alimento. }\end{array}$ \\
\hline
\end{tabular}

Fonte: Autores (2021).

Com a associação dos quatros artigos encontrado, a diarreia foi a consequência que mais prevaleceu, aparecendo nos 4 artigos, seguido de mortalidade infantil com 3 artigos, desnutrição com 3 artigos, morbidade com 3 artigos e problemas respiratórios agudos com 1 artigo, respectivamente.

Accioly, al. et (2017) evidenciou no estudo que a diarreia é uma consequência causada pelo desmame precoce. A imunoglobulina A (IgA), que é responsável pela formação da barreira da mucosa gastrointestinal, não consegue impedir a penetração de microrganismos, desse modo aumentando a incidência de diarreia. O desmame foi relacionado a uma elevação de 3 vezes nas taxas de hospitalizações ou morte associadas à diarreia Fawzy, et al. (2011).

Para os autores (Accioly, et al., Oliveira et al.) A introdução precoce de alimentos complementares, também, está associada ao aumento da morbidade e mortalidade infantil, devido à menor ingestão de anticorpos e imunoglobulinas, contidos no leite materno, além de proporcionar um maior risco de contaminação dos alimentos ofertados às crianças.

Um estudo realizado em Fortaleza, com 121 crianças de 0 a 10 anos de idade demostrou que 23,6\% das crianças com amamentação ausente ou ineficaz tiveram diarreia. O mesmo estudo mostrou que no estado do Ceará, a taxa de diarreia é de $24 \%$ no interior e de $16 \%$ na capital.

A prática do desmame precoce resulta também em maiores taxas de propensão a alergias alimentares. Essa associação é devida ao sistema digestivo e imunológico da criança serem ainda imaturos antes de completarem os seis meses de idade (José, et al.,2017). Um dos principais substitutos do leite materno nos primeiros meses de vida é o leite de vaca. Esta substituição resulta na exposição precoce do antígeno ao trato gastrointestinal imaturo do bebê, ocasionando casos alérgicos (Batista; Freitas; Haack, 2009).

De acordo com a Sociedade Brasileira de Pediatria (São Paulo, 2006), o desenvolvimento de alguma alergia alimentar está relacionado à quantidade e frequência da ingestão de proteínas alergênicas do leite de vaca como a caseína, nutriente de difícil digestão e excreção pelo organismo do lactente. Diante disso, o tempo de aleitamento materno diminuído interfere na absorção de nutrientes essenciais do leite materno e aumenta o risco de contaminação e de reações alérgicas (Santos et al, 2010; Monteiro et al, 2011). 
A introdução precoce da alimentação complementar à dieta dos lactentes está relacionada ao aparecimento de doenças alérgicas, asma, eczema e dermatite atópica, além de maior risco de desenvolvimento de aterosclerose e doenças crônicas não transmissíveis na vida adulta, tudo isso em decorrência da baixa absorção de nutrientes como ferro e zinco no período de lactação (Dias, et al., 2010; Arantes et al., 2011).

O aleitamento artificial interfere na realização das funções de mastigação, sucção e deglutição e pode levar à presença de alterações na musculatura orofacial, na postura de repouso dos lábios e da língua, alterações na formação da arcada dentária e alterações no palato

O desmame precoce está associado ao aumento do número de diarreia e outras infecções respiratórias porque diminui maturidade imunológica do bebê, prejudicando o desenvolvimento neuropsicomotor da criança e aumento de problemas dentários, síndrome do respirador bucal e distúrbios fono-articulatórios (Minas Gerais,2004)

A introdução precoce da alimentação complementar pode ocasionar danos a criança aumentando o risco de morbimortalidade, uma vez que a deixa mais suscetível ao desenvolvimento de diarreias, infecções respiratórias e gastrintestinais, e ainda desnutrição, comprometendo assim o crescimento e desenvolvimento da mesma (Dias, et al., 2010; Braga, et al., 2008). Outros danos incluem: sangramento intestinal clínico e subclínico, causando impacto negativo no estado nutricional do lactente que terá suas reservas de ferro diminuídas (Brasil, 2011).

A diarreia é considerada um grave problema de saúde pública, sendo a segunda causa de internação hospitalar infantil - precedida apenas pelas infecções respiratórias, consideradas a principal causa de mortalidade infantil. (Brasil. Ministério da Saúde).

Entre 2000 e 2010, foram notificados 29.491.078 casos de doenças diarreicas agudas no Brasil, sendo que somente a Região Norte apresentou, em 2006, 33 casos por 1.000 nascidos vivos e, em 2009, na Região Sudeste, a incidência foi de 15 casos por 1.000 nascidos vivos. (Brasil. Departamento de vigilância Epidemiológica).

Estima-se que 52,3\% das mortes por pneumonia em crianças possam ser atribuídas à desnutrição (27). A desnutrição proteico-energética pode afetar os mecanismos de defesa antígeno específicos e não-específicos. (Victora, Kirkwood, Ashworth, Black, Rogers, Sazawal, et al.).

Em um estudo inglês, a taxa média de internação por doenças respiratórias foi de 2.710 por 100.000 habitantes (Hawker et al., 2003).

No Brasil, as doenças infecciosas e parasitárias em 2009, foram a $4^{\mathrm{a}}$ causa de óbitos e $2^{\mathrm{a}}$ de hospitalizações no grupo etário de 0 a 4 anos. Desse grupo de doenças, as diarreicas são responsáveis por 1258 óbitos (Brasil. Sistema de Informação de Mortalidade. Mortalidade - Brasil). As crianças com maior risco de desenvolverem a doença são os lactentes e menores de 01 ano e mais suscetíveis ao quadro persistente, aumentando o tempo de hospitalização, óbitos e infecções sistêmicas (Façanha, Pinheiro).

Em termos de morbidade e mortalidade infantil, representam 2 bilhões de casos, matando 1,5 milhões de crianças anualmente em países subdesenvolvidos e em desenvolvimento. (WHO. World Health Organization).

Dados recentes apontam uma queda de 64\% na taxa de mortalidade infantil no Brasil, entre os anos de 1980 e 2006. A melhoria desse indicador, entre outros fatores, reflete o Programa de Assistência Integral à Saúde da Criança (PAISC), criado em 1984, tendo como princípio promover ações específicas na assistência à saúde infantil, com enfoque nas ações básicas de saúde, pretendendo assim reduzir a morbimortalidade infantil. Instituto Brasileiro de Geografia e Estatística, Ministério da Saúde).

Segundo dados do Ministério da Saúde, no Brasil a taxa de mortalidade infantil vem apresentado queda significativa, embora ainda seja alta. A taxa de mortalidade infantil apresentou, em média, uma redução de 59\% entre 1997 e 2007, indo de 
47,1 para 19,3 para cada mil nascidos vivos. A taxa de mortalidade em São Paulo já alcançou valores considerados baixos, sendo, em 2016, 12,90 para cada 1000 nascidos vivos.

Desde 1980, com a criação da Meta do Milênio houve uma redução da morbimortalidade infantil e diarreia. A taxa de mortalidade infantil (menores de 1 ano) por mil nascidos vivos passou de 29,7, em 2000, pra 15,6, em 2010. Essa taxa é menor que a meta prevista para 2015, de 15,7 por mil nascidos vivos. A queda mais acentuada ocorreu na região Norte (-58,6\%), que ainda apresenta a taxa mais elevada do Brasil:18,5 por mil nascidos vivos.

A taxa de mortalidade das crianças abaixo de cinco anos apresentou queda de 65\% entre 1990 e 2010 . O número de óbitos por mil nascidos vivos passou de 53,7 para 19 óbitos. Os indicadores demonstram que tanto as taxas de mortalidade na infância (menores de 5 anos) e infantil (menores de 1 ano) apresentaram forte queda entre 1990 e 2010 . A mortalidade infantil está concentrada nos primeiros meses de vida, no período neonatal precoce ( 0 a 6 dias) e neonatal tardio ( 7 a 27 dias).

O Brasil é uma das nações que têm se destacado por reduzir a mortalidade infantil e na infância. Entre 1990 e 2015, a taxa de mortalidade de crianças de até 1 ano caiu 73,67\%(Sinasc). Globalmente, 2,6 milhões de recém-nascidos morreram em 2016 - ou 7.000 todos os dias. As mortes neonatais representaram $46 \%$ de todas as mortes menores de cinco, aumentando de 41\% em 2000. Taxa de mortalidade neonatal caiu 49\%, de 37 óbitos por 1.000 nascidos vivos em 1990 a 19 em 2016. (Unicef, 2017).

\section{Considerações Finais}

Baseado nos resultados encontrados, o aleitamento materno é importante tanto para prevenção de doenças infecciosas, quanto para o crescimento e desenvolvimento da criança. As crianças desmamadas precocemente podem apresentar, diarreia, infecções respiratórias agudas, desnutrição, morbidade e mortalidade infantil obesidade, desenvolvimento motor-oral incompleto e alergias. É necessário criar estratégias para que incentive as mães amamentarem os lactentes até completarem seis meses de vida. Algumas estratégias adotadas pode ser: orientar as mães a ordenhar e armazenar o leite materno nesse período que elas precisam se ausentar. As empresas oferecerem berçários para que as mães consigam amamentar as crianças. E o empregador aumentar o tempo da licença maternidade.

\section{Referências}

Brown, A., et al. A descriptive study investigating the use and nature of baby-led weaning in a UK sample of mothers. BVS.

Brown, A. Differences in eating behaviour, well-being and personality between mothers following baby-led vs. traditional weaning styles. $B V S$.

Caleyachetty, A., et al. Breastfeeding duration, age of starting solids and high BMI risk and adiposity in Indian children. BVS.

Feitosa, M. E. B. et al. Breastfeeding: causes and consequences of early weaning. Research, Society and Development, 9(7), e856975071. 10.33448/rsdv9i7.5071.

Magnusson, M., et al. No widening socioeconomic gap within a general decline in Swedish breastfeeding. BVS.

Monteiro, J. R. S., et al. Fatores associados a interrupção precoce do aleitamento materno exclusivo em prematuros. BVS.

Nunes, H. J. M., et al. Causas e consequências do desmame precoce e as intervenções dos profissionais enfermeiros. Revista Ciência \& Saberes-UniFacema, 4(3).

Rogers, N. L., et al. Colostrum avoidance, prelacteal feeding and late breast-feeding initiation in rural Northern Ethiopia. BVS.

Silva, D., et al. Aleitamento materno: causas e consequências do desmame precoce. Unimontes Científica, 19(2), $146-157$.

Silva, J. N. Aleitamento Materno: Motivos E Consequências Do Desmame Precoce Em Crianças. Revista Artigos. Com, 20 , e4756.

Taha, T. E., et al. Effects of cessation of breastfeeding in HIV-1-exposed, uninfected children in Malawi. BVS.

Taveiro, E. A. N., et al. Adesão ao Aleitamento Materno Exclusivo em Bebês de 0 a 6 Meses Nascidos em um Hospital e Maternidade do Município de São Paulo. BVS.

Vazir, S., et al. Cluster-randomized trial on complementary and responsive feeding education to caregivers found improved dietary intake, growth and development among rural Indian toddlers. BVS. 